$$
\text { ङ }
$$

\title{
Two distinct kinetic regimes for the relaxation of light-induced superconductivity in $\mathrm{La}_{1.675} \mathrm{Eu}_{0.2} \mathrm{Sr}_{0.125} \mathrm{CuO}_{4}$
}

\author{
C. R. Hunt, ${ }^{1,2}$ D. Nicoletti, ${ }^{1}$ S. Kaiser, ${ }^{1}$ T. Takayama, ${ }^{3,4}$ H. Takagi, ${ }^{3,4,5}$ and A. Cavalleri ${ }^{1,6}$ \\ ${ }^{1}$ Max Planck Institute for the Structure and Dynamics of Matter, Hamburg, Germany \\ ${ }^{2}$ Department of Physics, University of Illinois at Urbana-Champaign, Urbana, Illinois 61801, USA \\ ${ }^{3}$ Department of Advanced Materials Science, University of Tokyo, Tokyo, Japan \\ ${ }^{4}$ Max-Planck-Institut für Festkörperforschung, Heisenbergstrasse 1, D-70569 Stuttgart, Germany \\ ${ }^{5}$ RIKEN Advanced Science Institute, Hirosawa 2-1, Wako 351-0198, Japan \\ ${ }^{6}$ Department of Physics, Oxford University, Clarendon Laboratory, Oxford, United Kingdom
}

(Received 16 October 2014; revised manuscript received 29 December 2014; published 29 January 2015;

publisher error corrected 30 January 2015)

\begin{abstract}
We address the kinetic competition between charge striped order and superconductivity in $\mathrm{La}_{1.675} \mathrm{Eu}_{0.2} \mathrm{Sr}_{0.125} \mathrm{CuO}_{4}$. Ultrafast optical excitation is tuned to a midinfrared vibrational resonance that destroys charge order and promptly establishes transient coherent interlayer coupling in this material. This effect is evidenced by the appearance of a longitudinal plasma mode reminiscent of a Josephson plasma resonance. We find that coherent interlayer coupling can be generated up to the charge-order transition $T_{\mathrm{CO}} \approx 80 \mathrm{~K}$, far above the equilibrium superconducting transition temperature of any single layer cuprate. Two key observations are extracted from the relaxation kinetics of the interlayer coupling. First, the plasma mode relaxes through a collapse of its coherence length and not its density. Second, two distinct kinetic regimes are observed for this relaxation, above and below spin-order transition $T_{\mathrm{SO}} \approx 25 \mathrm{~K}$. In particular, the temperature-independent relaxation rate observed below $T_{\mathrm{SO}}$ is anomalous and suggests coexistence of superconductivity and stripes rather than competition. Both observations support arguments that a low temperature coherent stripe (or pair density wave) phase suppresses $c$-axis tunneling by disruptive interference rather than by depleting the condensate.
\end{abstract}

Stripe order in cuprates is closely associated with the suppression of superconductivity, although the interplay between these orders remains a subject of much debate. In these materials, superconductivity is thought to be supported in two-dimensional $\mathrm{CuO}_{2}$ planes and made three dimensional by interlayer Josephson tunneling. However, both in-plane and out-of-plane coherence can be strongly affected by ordering of charges and spins, or by lattice deformations. Small perturbations in doping, applied field, or pressure can tune the energy landscape between orders, suppressing or supporting the superconducting state.

This phase competition is especially dramatic in the lanthanum copper oxides, which exhibit "striped" spin and charge-ordered states, typically stabilized by an underlying lattice distortion [1,2]. The charge and spin orders also organize within the $\mathrm{CuO}_{2}$ planes. Static stripe orders, first discovered in $\mathrm{La}_{1.6-x} \mathrm{Nd}_{0.4} \mathrm{Sr}_{x} \mathrm{CuO}_{4}$ (LNSCO $x$ ) [3], are also found in $\mathrm{La}_{2-x} \mathrm{Ba}_{x} \mathrm{CuO}_{4}$ (LBCO $x$ ) [4,5] and $\mathrm{La}_{1.8-x} \mathrm{Eu}_{0.2} \mathrm{Sr}_{x} \mathrm{CuO}_{4}$ $(\operatorname{LESCO} x)$ [6,7] [see phase diagram in Fig. 1(a)]. Charge stripes are associated with the suppression of superconductivity in this family of compounds, with bulk superconductivity completely destroyed at $x=1 / 8$ doping [8,9] where the lattice spacing synchronizes with the stripe periodicity.

Recent theoretical [10-13] and experimental $[9,14,15]$ work suggests that this suppression is not a simple competition between charge density wave and superconducting instabilities. Rather, superconductivity may coexist with charge ordering, which imposes a space dependence on the superconducting order parameter phase [11]. This spatial modulationoften referred to as a "pair density wave" state—suppresses the total Josephson tunneling by disruptive interference [12]. According to this view, three-dimensional superconductivity would only be achieved below the temperature at which the superconducting $c$-axis coherence length exceeds the quadrupled unit cell spacing [16] [see inset in Fig. 1(a)]. This assessment is supported by susceptibility [9] and resistivity and thermopower [15] measurements that suggest a fluctuating two-dimensional (2D) superconductivity regime survives in the spin-order state, up to $T_{\mathrm{SO}}=40 \mathrm{~K}$ in $\mathrm{LBCO} 1 / 8$, and one-dimensional correlations persist up to the charge-order transition, $T_{\mathrm{CO}}=54 \mathrm{~K}$.

In this work, we used femtosecond laser excitation to reintroduce $c$-axis coherent coupling in stripe-ordered $\mathrm{La}_{1.675} \mathrm{Eu}_{0.2} \mathrm{Sr}_{0.125} \mathrm{CuO}_{4}$ (LESCO 1/8) and examined the relaxation kinetics. LESCO $1 / 8$ single crystals were grown using the traveling solvent floating zone technique. They were characterized by resistivity and magnetization measurements and were found to be nonsuperconducting down to $5 \mathrm{~K}$. [17] The charge- and spin-ordered regimes for this doping are indicated by the dashed vertical line on the phase diagram in Fig. 1(a).

Laser excitation has proven a powerful tool to control lattice [18-21] and electronic [22-25] properties and drive phase transitions. The $15 \mu \mathrm{m}$ midinfrared (MIR) pump used here, tuned to an in-plane $\mathrm{Cu}-\mathrm{O}$ mode, has been shown to promptly reduce charge stripe order [26] and reintroduce $c$-axis coherent coupling [17]. The $c$-axis optical response below $2.6 \mathrm{THz}$ was interrogated as a function of time delay after photoexcitation. Single-cycle THz pulses were generated via $800 \mathrm{~nm}$ excitation of a photoconductive antenna. The time resolution of the experiment was limited by the $\mathrm{THz}$ bandwidth to about $300 \mathrm{fs}$. The experiment was performed in reflection geometry, with the MIR pump at normal incidence and the $\mathrm{THz} s$-polarized along the $c$ axis at a $30^{\circ}$ angle of incidence. The reflected equilibrium 
(a)
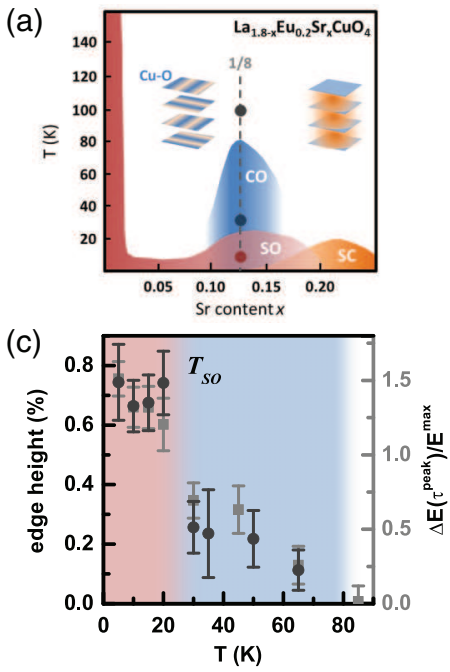

FIG. 1. (Color online) Phase diagram of LESCO and transient response at 5, 30, and $100 \mathrm{~K}$. (a) Phase diagram of LESCO, based on Ref. [28] indicating regions of bulk superconductivity (SC) and static spin ( $\mathrm{SO}$ ) and charge (CO) order. The static stripes suppress $c$-axis coupling of the $\mathrm{CuO}_{2}$ planes (inset cartoon, left), with bulk superconductivity restored at dopings in which the stripe order is reduced (inset cartoon, right). (b) The raw transient reflectivity changes measured $1.8 \mathrm{ps}$ after MIR excitation. At $5 \mathrm{~K}$ (b.1) and $30 \mathrm{~K}$ (b.2), an edge is apparent at 1.5-2 THz. The black lines indicate the reflectivity spectrum (rescaled) due to a longitudinal plasma mode, shown as a guide to the eye. Above the charge-ordered transition temperature $T_{\mathrm{CO}}$ no edge is observed [shown at $100 \mathrm{~K}$; (b.3)]. (c) The size of the reflectivity edge remains approximately constant below the spin-order transition temperature $T_{\mathrm{SO}}$, but drops rapidly between $T_{\mathrm{SO}}$ and $T_{\mathrm{CO}}$ (dark gray circles). This trend is also reflected in the time domain, by the change in the THz amplitude $\Delta E(\tau) / E^{\text {max }}$ measured at the peak of the response (light gray squares). The full delay traces $\Delta E(\tau) / E^{\max }$ are shown in Fig. 4.
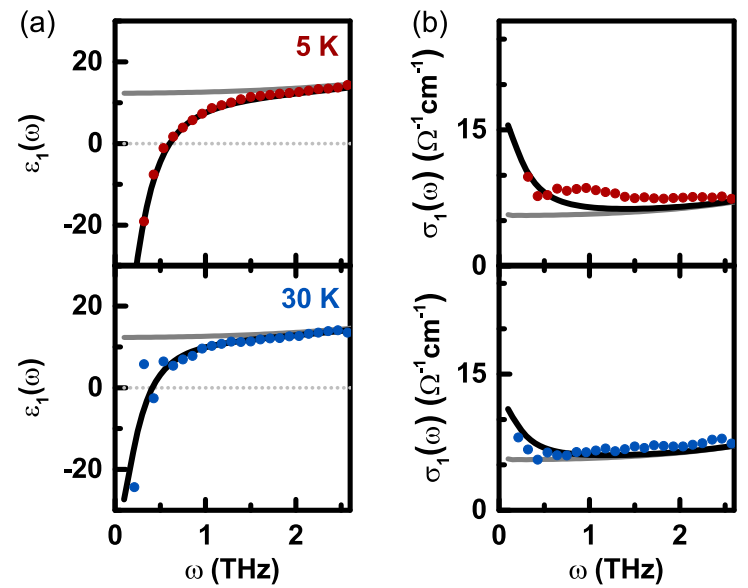

THz field, $E(t)$, and the pump-induced changes to the field at time delay $\tau, \Delta E(t, \tau)$, were measured via electro-optic sampling to capture the full amplitude and phase response.

The raw change in reflectivity, given by the Fourier transformed quantity $|\Delta \tilde{E}(\omega, \tau) / \tilde{E}(\omega)| \equiv \Delta R / R$, is shown in Fig. 1(b) at $\tau=1.8 \mathrm{ps}$ after excitation, which corresponds to the peak amplitude of the transient response. Below the charge-order transition temperature, the transient spectra show the appearance of a reflectivity edge between 1.5 and $2 \mathrm{THz}$. Figure 1(b.1) shows $5 \mathrm{~K}$ base temperature and Fig. 1(b.2) shows $30 \mathrm{~K}$, above $T_{\mathrm{SO}}$. Note that the appearance of this effect has been reported in Ref. [17] up to 10-20 K. Improvements in the experimental apparatus and a fourfold increase in the excitation fluence (to $\sim 4 \mathrm{~mJ} / \mathrm{cm}^{2}$ ) make it possible here to detect a qualitatively similar but smaller edge far above $T_{\text {So }}$, up to between 70 and $80 \mathrm{~K}$ [dark gray dots, Fig. 1(c)]. Above $T_{\mathrm{CO}}$, the $\mathrm{THz}$ response shows no reflectivity edge [Fig. 2(b.3) at $100 \mathrm{~K}$ ].

The complex optical properties of the photoinduced state were calculated from the $\mathrm{THz}$ response and equilibrium properties measured by Fourier transform infrared (FTIR) spectroscopy. The $\mathrm{THz}$ probe samples a crystal volume on order 100 times greater than the $15 \mu \mathrm{m}$ pump. To isolate the optical response of the photoexcited volume alone we model the system as a single excited layer on a bulk volume that remains in equilibrium [27] (see Supplemental Material [28]).

Figure 2 shows the optical response of LESCO 1/8 at two temperatures, below and above the spin-order transition $T_{\mathrm{SO}} \approx 25 \mathrm{~K}$. [6,29] In both regimes, the transient response (dots) is characterized by the appearance of a longitudinal plasma mode at $\sim 1 \mathrm{THz}$. This mode is most clearly discerned in the real dielectric response $\varepsilon_{1}(\omega)$, shown in Fig. 2(a), which exhibits a zero crossing near the mode resonance. The Ohmic conductivity $\sigma_{1}(\omega)$ is only weakly affected by the pump, maintaining an insulatorlike response [Fig. 2(b)], suggesting the pump is not producing significant quasiparticle excitations. The inductive conductivity, $\sigma_{2}(\omega)$, which is negative in equilibrium (gray line) and approaches zero as
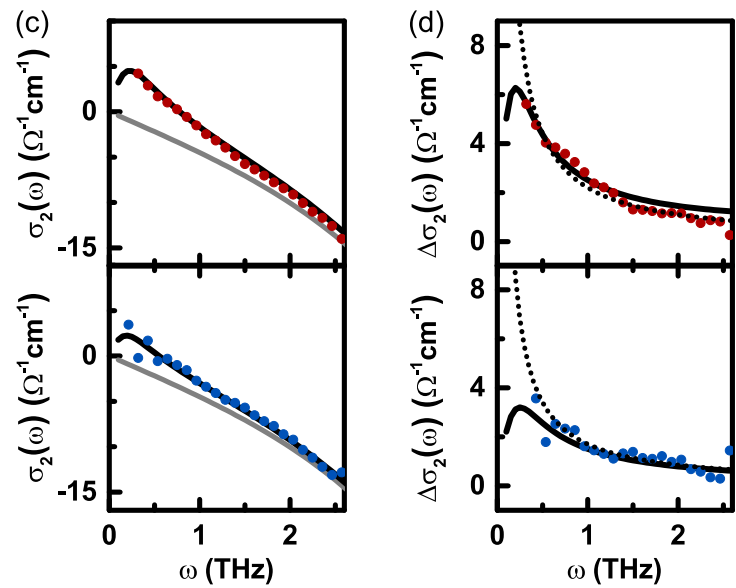

FIG. 2. (Color online) Transient optical properties at 5 and $30 \mathrm{~K}$. Upon MIR excitation, LESCO 1/8 develops a high-mobility state, shown 2.8 ps after excitation at $5 \mathrm{~K}$ (red, first row) and at the peak of the response at $30 \mathrm{~K}$ (blue, second row). The equilibrium response is shown in gray. The transient state can be fit (black lines) by a longitudinal plasma mode, as described in the main text. (a) The mode is characterized by a zero crossing in the real dielectric function, $\varepsilon_{1}(\omega)$. (b) The Ohmic conductivity $\sigma_{1}(\omega)$ remains insulating, showing only a small enhancement at lowest frequencies. (c) The inductive conductivity $\sigma_{2}(\omega)$ becomes positive and diverging towards low frequency. (d) The pump-induced changes $\Delta \sigma_{2}(\omega)=\sigma_{2}(\omega)-\sigma_{2}^{\text {eq }}(\omega)$ diverge as $1 / \omega$ (dotted line). 
$\omega \rightarrow 0$, instead increases divergently towards low frequency in the photoexcited state [Fig. 2(c)]. The response turns positive below $0.75 \mathrm{THz}$ at $5 \mathrm{~K}$ and $0.5 \mathrm{THz}$ at $30 \mathrm{~K}$. The conductivity change, $\Delta \sigma_{2}(\omega)=\sigma_{2}(\omega)-\sigma_{2, \text { eq }}(\omega)$, scales as $1 / \omega$, as shown by the dotted line in Fig. 2(d).

In equilibrium, LESCO $1 / 8$ has no plasma mode in this frequency range at any temperature. However, the transient mode coincides with the frequency of the Josephson plasma resonance (JPR) of related underdoped superconducting lanthanides [14,30]. A Josephson plasma mode is a generic feature of superconductivity in cuprates which arises due to the tunneling of pairs between $\mathrm{CuO}_{2}$ planes. Its frequency $\omega_{p}$ is related to the condensate density, compressibility, and the geometric spacing of the planes [31]. Within one family of compounds, the JPR frequency scales with the superfluid density as $\omega_{\mathrm{JPR}}^{2} \tilde{\propto} \mathrm{n}_{\mathrm{SF}}$, implying that the transient state reported here has a condensate density of roughly half that of nearoptimal doped $\mathrm{La}_{1.85} \mathrm{Sr}_{0.15} \mathrm{CuO}_{4}$ in equilibrium.

Hence, although the appearance of a longitudinal plasma mode by itself does not uniquely prove superconductivity, the quantitative agreement with the optical response of related superconducting compounds makes the assignment of a superconducting state by far the most likely explanation.

The solid black lines in Fig. 2 show a fit to a single longitudinal plasma mode utilizing only two free parameters [32] with the Drude form,

$$
\tilde{\varepsilon}=\tilde{\varepsilon}_{\text {eq }}-\frac{\omega_{p}^{2}}{\omega^{2}-i \omega \Gamma},
$$

where $\tilde{\varepsilon}_{\text {eq }}$ is the equilibrium dielectric function. The transient response is best fit by $\omega_{p}=2.45 \mathrm{THz}(1.65 \mathrm{THz})$ at $5 \mathrm{~K}(30 \mathrm{~K})$ with a scattering rate of $\Gamma \approx 0.25 \mathrm{THz}$.

Within this model, the scattering rate term $\Gamma$ encompasses all transient processes that impact the mobility along the $c$ axis. The scattering rate is extremely low, and cannot be reconciled with the properties of an incoherent plasma, but is consistent with a superconducting state with finite coherence length such as that seen in the equilibrium superconductor $\mathrm{La}_{2-x} \mathrm{Sr}_{x} \mathrm{CuO}_{4}$ near $T_{c}$ [33]. At resonance, transport occurs across the $\mathrm{CuO}_{2}$ planes with a velocity $2 \omega_{P} L$, where $L$ is the $\mathrm{CuO}_{2}$ plane separation. The rate $\Gamma$ can be related to the coherence length $d$ of the $c$-axis plasma by $d=2 \omega_{P} L / \Gamma$.

Note that the reflectivity edge and the zero crossing of $\varepsilon_{1}(\omega)$ do not appear exactly at the plasma resonance, $\omega_{P}$, but are shifted due to decoherence as well as other intraband contributions to $\varepsilon_{1 \text {,eq }}$, which can be captured in the $\mathrm{THz}$ regime by a single parameter $\varepsilon_{\mathrm{FIR}}$. For long coherence lengths, $d \rightarrow$ $\infty$, the zero crossing occurs near the screened frequency $\tilde{\omega}_{P}=$ $\omega_{P} / \sqrt{\varepsilon_{\mathrm{FIR}}}$ and shifts to the red as $d$ decreases. If $\Gamma>\tilde{\omega}_{P}$, the zero crossing is entirely lifted. For the plasma mode $\tilde{\omega}_{P}$ reported here, we take $\varepsilon_{\mathrm{FIR}}=30$, a standard value for cuprates [34,35].

Figure 3 shows the evolution of $\varepsilon_{1}(\omega)$ as a function of delay time after photoexcitation at $5 \mathrm{~K}$ [Fig. 3(a.1)], $35 \mathrm{~K}$ [Fig. 3(a.2)], and $65 \mathrm{~K}$ [Fig. 3(a.3)]. From fits to the optical response, we extract the time evolution of the screened plasma mode $\tilde{\omega}_{P}$ [Fig. 3(b), bottom] and the coherence length $d$ [Fig. 3(b), top]. After the transient state is formed, both quantities initially decay following a 2 ps (1 ps) time scale at $5 \mathrm{~K}(35 \mathrm{~K})$. At longer time delays, the plasma frequency
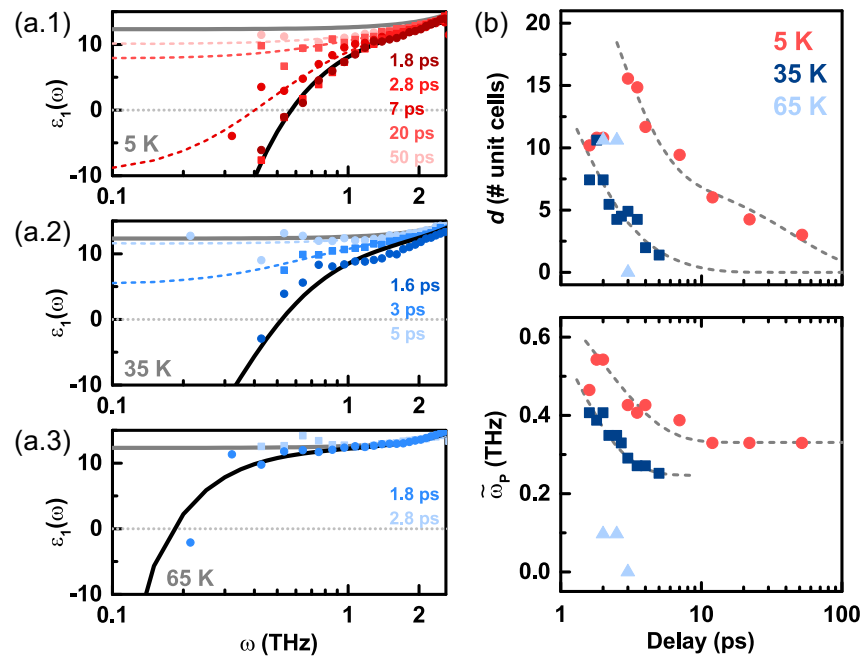

FIG. 3. (Color online) Time dependence of the transient response. (a) The real dielectric function $\varepsilon_{1}(\omega)$ (alternating circle and square dots) as a function of pump-probe delay at $5 \mathrm{~K}$ (a.1), $35 \mathrm{~K}$ (a.2), and $65 \mathrm{~K}$ (a.3). The response is fit with a single longitudinal mode, as described in the main text (solid black and dashed lines). The drop in coherence length causes a flattening in the low frequency $\varepsilon_{1}(\omega)$. (b, top) The $c$-axis coherence length of the transient plasma as a function of delay for three temperatures: $5 \mathrm{~K}$ (circles), $35 \mathrm{~K}$ (squares), and $65 \mathrm{~K}$ (triangles). The length is expressed in units of the $\mathrm{CuO}_{2}$ plane spacing. The decay can be fit with a double exponential (dashed lines) with time constants $2 \mathrm{ps}(1 \mathrm{ps})$ and $45 \mathrm{ps}(4 \mathrm{ps})$ at $5 \mathrm{~K}$ $(35 \mathrm{~K})$. (b, bottom) The plasma frequency $\omega_{P}$ redshifts at early times, decaying to a constant value following single exponential (dashed lines) of $2 \mathrm{ps}(1 \mathrm{ps})$ at $5 \mathrm{~K}(35 \mathrm{~K})$.

stabilizes to a finite value, indicating that the carrier density does not reduce significantly throughout the relaxation. This observation offers further support that the transient state is not consistent with an anomalously high-mobility quasiparticle excitation, which would relax via a depletion of the carrier density. Rather, the decay of the plasma mode is characterized by a dramatic decrease in the correlation length from $\sim 15$ unit cells $(10 \mathrm{~nm})$ to zero.

The highest temperature at which the longitudinal mode could be seen in $\varepsilon_{1}(\omega)$ in our frequency window was $65 \mathrm{~K}$. The low-frequency cutoff is limited by the sample size and by day-to-day alignment to between 0.2 and $0.4 \mathrm{GHz}$. From the optical response, and the amplitude of the reflectivity changes $\Delta E(\tau) / E^{\max }$, we extrapolate that the maximum base temperature at which the transient state can be induced is between 70 and $80 \mathrm{~K}$, which is approximately the chargeordering temperature $T_{\mathrm{CO}}$.

The relaxation time scales are also captured by the changes in reflectivity measured at the peak of the $\mathrm{THz}$ field, $\Delta E(\tau) / E^{\max }$, which could be measured with finer delay steps. Figure 4(a) shows $\Delta E(\tau) / E^{\max }$ as a function of pump-probe delay $\tau$. Two lifetimes could be extracted, shown in blue in Fig. 4(b). The lifetimes measured from the coherence length decay are shown in red for comparison. In both the spin-ordered and charge-ordered regimes, the time scales of the double exponential decay are commensurate with the decay of the coherence length. Both lifetimes exhibit two distinct 

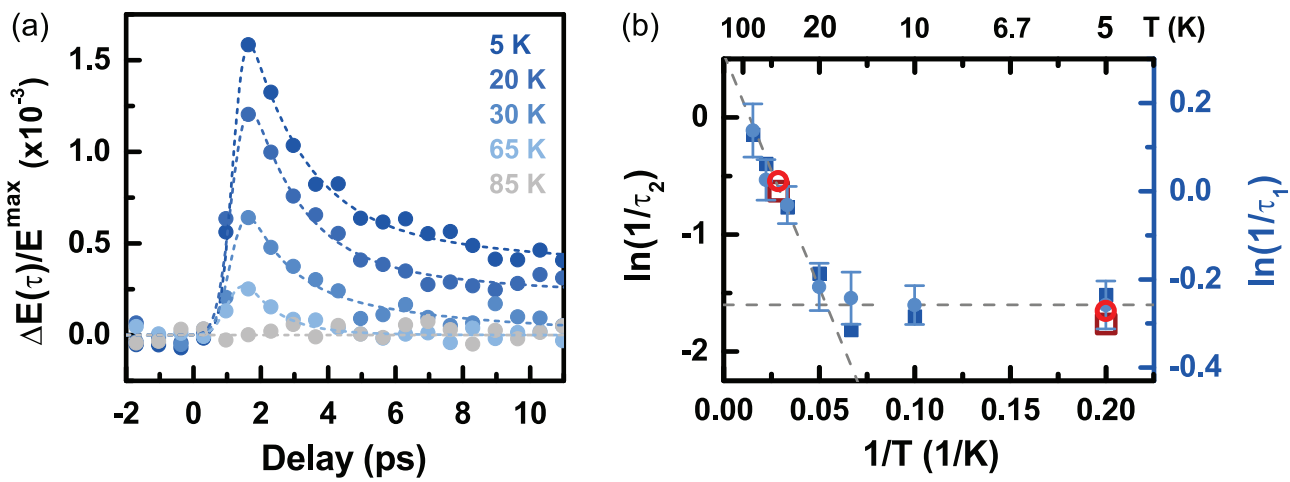

FIG. 4. (Color online) Temperature dependence of the transient lifetime. (a) The time profile of the transient state as a function of pump-probe delay. The vertical axis indicates the transient change of the peak of the THz probe field. For clarity, each point shown represents an average of ten delay measurements. The lifetime of the transient state was extracted using a double exponential fit (dashed lines), with each exponential given roughly equal weight, $50 \% \pm 10 \%$. (b) An Arrhenius plot of the relaxation rate as a function of temperature. The short lifetime $\tau_{1}$ (dark blue squares) and the long lifetime $\tau_{2}$ (light blue circles) track the decay of the coherence length of the transient plasma. [Lifetimes used in the fits shown in Fig. 3(b) are plotted in red.] The lifetime of the transient state remains temperature independent (horizontal gray dashed line) below the spin-order transition temperature, $T_{\mathrm{SO}} \approx 25 \mathrm{~K}$. Above this transition, the lifetime exhibits an exponential temperature dependence, with an energy scale of $4 \mathrm{meV}$ for $\tau_{2}$ and $0.8 \mathrm{meV}$ for $\tau_{1}$ (gray dashed line).

regimes. Below $T_{\mathrm{SO}}$, the lifetimes remain temperature independent. Above $T_{\mathrm{SO}}$, where only static charge order remains, the lifetime drops exponentially with base temperature.

The exponential dependence of the relaxation between $T_{\mathrm{SO}}<T<T_{\mathrm{CO}}$ can be reconciled with the expected kinetic behavior for a transition between two distinct thermodynamic phases separated by a free energy barrier. This is quantitatively captured by the slope of the logarithmic plot in Fig. 4(b), which reflects an activated relaxation of the type $\exp \left(-E_{\text {barrier }} / k_{B} T\right)$. From a double exponential relaxation with lifetimes $\tau_{1}$ and $\tau_{2}$, we extract an energy scale $E_{\text {barrier }} \sim 40 \mathrm{~K}(4 \mathrm{meV})$ and $9 \mathrm{~K}$ $(0.8 \mathrm{meV})$, respectively. Interestingly, this energetic regime corresponds to the energy scale of spin fluctuations measured in LSCO 1/8 [36-38], suggesting that the transition between the two phases may be regulated by spin rearrangements. This is consistent with the observation that strong spin fluctuations develop in equilibrium above $T_{\mathrm{SO}}$ and survive up to $T_{\mathrm{CO}}$ $[39,40]$.

The departure from activated behavior for $T<T_{\mathrm{SO}}$ may therefore be related to the freezing out of spin fluctuations. A temperature-independent relaxation rate would be compatible with quantum coherent tunneling between two states, for instance between superconducting and pair density wave phases at constant carrier density. In this picture, Cooper pairing would be superimposed or intertwined [41-43] with the stripe phase, where the dynamical destruction of stripes allows a finite Josephson current rather than driving pairing directly. Indeed, as discussed in Ref. [17], the prompt time scale of the appearance of the longitudinal mode renders it unlikely that the optical excitation is causing pair formation
[44] but rather suggests that pairing in the planes persists in equilibrium.

We have reported the generation of a transient high-mobility state in LESCO 1/8 that survives up to the charge-order transition. This transient state is characterized by the appearance of a longitudinal plasma mode in the low-frequency $\mathrm{THz}$ response, which we attribute to $c$-axis superconducting coupling. A first striking observation is that the transient plasma mode can be induced all the way up to the charge-order transition temperature $T_{\mathrm{CO}} \sim 80 \mathrm{~K}$, a large temperature for this family of cuprates. This observation further substantiates theoretical ideas that charge-ordered cuprates remain frustrated superconductors above their equilibrium transition temperature, and that pairing persists even above the spin-ordering temperature $T_{\text {So }}$. We find that two distinct regimes of phase competition exist between stripe order and transient superconductivity. The lifetime of the photoinduced state remains essentially independent of base temperature below $T_{\mathrm{SO}}$ and follows an Arrhenius-like behavior in the charge-ordered regime, up to $T_{\mathrm{CO}}$. Our measurements support the growing body of evidence for $2 \mathrm{D}$ superconducting pairing in the charge-ordered state of cuprates.

C.R.H., D.N., S.K., and A.C. acknowledge funding support from the European Research Council under the European Union's Seventh Framework Programme (FP7/20072013)/ERC Grant Agreement No. 319286. T.T. and H.T. acknowledge funding support from the Japanese Ministry of Education, Culture, Sports, Science and Technology (MEXT) Grant-in-Aid for Scientific Research (S) (19104008).
[1] Takashi Suzuki and Toshizo Fujita, Structural phase transition in $\left(\mathrm{La}_{1-x} \mathrm{Ba}_{x}\right)_{2} \mathrm{CuO}_{4-\delta}$, Physica C 159, 111 (1989).

[2] M. K. Crawford, R. L. Harlow, E. M. McCarron, W. E. Farneth, J. D. Axe, H. Chou, and Q. Huang, Lattice instabilities and the effect of copper-oxygen-sheet distortions on superconductivity in doped $\mathrm{La}_{2} \mathrm{CuO}_{4}$, Phys. Rev. B 44, 7749 (1991).

[3] J. M. Tranquada, B. J. Sternlieb, J. D. Axe, Y. Nakamura, and S. Uchida, Evidence for stripe correlations of spins and holes 
in copper oxide superconductors, Nature (London) 375, 561 (1995).

[4] M. Fujita, H. Goka, K. Yamada, and M. Matsuda, Competition between charge- and spin-density-wave order and superconductivity in $\mathrm{La}_{1.875} \mathrm{Ba}_{0.125-x} \mathrm{Sr}_{x} \mathrm{CuO}_{4}$, Phys. Rev. Lett. 88, 167008 (2002).

[5] P. Abbamonte, A. Rusydi, S. Smadici, G. D. Gu, G. A. Sawatzky, and D. L. Feng, Spatially modulated "Mottness" in $\mathrm{La}_{2-x} \mathrm{Ba}_{x} \mathrm{CuO}_{4}$, Nat. Phys. 1, 155 (2005).

[6] H. H. Klauss, W. Wagener, M. Hillberg, W. Kopmann, H. Walf, F. J. Litterst, M. Hucker, and B. Buchner, From antiferromagnetic arder to static magnetic stripes: The phase diagram of $(\mathrm{La}, \mathrm{Eu})_{2-x} \mathrm{Sr}_{x} \mathrm{CuO}_{4}$, Phys. Rev. Lett. 85, 4590 (2000).

[7] M. Hücker, G. D. Gu, J. M. Tranquada, M. von Zimmermann, H. H. Klauss, N. J. Curro, M. Braden, and B. Buchner, Coupling of stripes to lattice distortions in cuprates and nickelates, Physica C 460-462, 170 (2007).

[8] A. R. Moodenbaugh, Youwen Xu, M. Suenaga, T. J. Folkerts, and R. N. Shelton, Superconducting properties of $\mathrm{La}_{2-x} \mathrm{Ba}_{x} \mathrm{CuO}_{4}$, Phys. Rev. B 38, 4596 (1988).

[9] M. Hücker, M. v. Zimmermann, G. D. Gu, Z. J. Xu, J. S. Wen, Guangyong Xu, H. J. Kang, A. Zheludev, and J. M. Tranquada, Stripe order in superconducting $\mathrm{La}_{2-x} \mathrm{Ba}_{x} \mathrm{CuO}_{4}(0.095 \leqslant x \leqslant$ 0.155), Phys. Rev. B 83, 104506 (2011).

[10] S.-C. Zhang, Recent developments in the $\mathrm{SO}(5)$ theory of high $T_{c}$ superconductivity, J. Phys. Chem. Solids 59, 1774 (1998).

[11] A. Himeda, T. Kato, and M. Ogata, Stripe states with spatially oscillating $d$-wave superconductivity in the two-dimensional $t-t^{\prime}-J$ model, Phys. Rev. Lett. 88, 117001 (2002).

[12] E. Berg, E. Fradkin, E.-A. Kim, S. A. Kivelson, V. Oganesyan, J. M. Tranquada, and S. C. Zhang. Dynamical layer decoupling in a stripe-ordered high- $T_{c}$ superconductor, Phys. Rev. Lett. 99, 127003 (2007).

[13] E. Berg, E. Fradkin, S. A. Kivelson, and J. M. Tranquada, Striped superconductors: How spin, charge and superconducting orders intertwine in the cuprates, New J. Phys. 11, 115004 (2009).

[14] D. Nicoletti, E. Casandruc, Y. Laplace, V. Khanna, C. R. Hunt, S. Kaiser, S. S. Dhesi, G. D. Gu, J. P. Hill, and A. Cavalleri. Optically induced superconductivity in striped $\mathrm{La}_{2-x} \mathrm{Ba}_{x} \mathrm{CuO}_{4}$ by polarization-selective excitation in the near infrared, Phys. Rev. B 90, 100503(R) (2014).

[15] Q. Li, M. Hücker, G. D. Gu, A. M. Tsvelik, and J. M. Tranquada, Two-dimensional superconducting fluctuations in stripe-ordered $\mathrm{La}_{1.875} \mathrm{Ba}_{0.125} \mathrm{CuO}_{4}$, Phys. Rev. Lett. 99, 067001 (2007).

[16] M. v. Zimmermann, A. Vigliante, T. Niemoeller, N. Ichikawa, T. Frello, J. Madsen, P. Wochner, S. Uchida, N. H. Andersen, J. M. Tranquada, D. Gibbs, and J. R. Schneider, Hard-x-ray diffraction study of charge stripe order in $\mathrm{La}_{1.48} \mathrm{Nd}_{0.4} \mathrm{Sr}_{0.12} \mathrm{CuO}_{4}$, Europhys. Lett. 41, 629 (1998).

[17] D. Fausti, R. I. Tobey, N. Dean, S. Kaiser, A. Dienst, M. C. Hoffmann, S. Pyon, T. Takayama, H. Takagi, and A. Cavalleri, Light-induced superconductivity in a stripe-ordered cuprate, Science 331, 189 (2011).

[18] M. Rini, R. Tobey, N. Dean, J. Itatani, Y. Tomioka, Y. Tokura, R. W. Schoenlein, and Andrea Cavalleri, Control of the electronic phase of a manganite by mode-selective vibrational excitation, Nature (London) 449, 72 (2007).

[19] M. Först, R. I. Tobey, S. Wall, H. Bromberger, V. Khanna, A. L. Cavalieri, Y.-D. Chuang, W. S. Lee, R. Moore, W. F. Schlotter,
J. J. Turner, O. Krupin, M. Trigo, H. Zheng, J. F. Mitchell, S. S. Dhesi, J. P. Hill, and A. Cavalleri, Driving magnetic order in a manganite by ultrafast lattice excitation, Phys. Rev. B 84, 241104(R) (2011).

[20] K. W. Kim, A. Pashkin, H. Schäfer, M. Beyer, M. Porer, T. Wolf, C. Bernhard, J. Demsar, R. Huber, and A. Leitenstorfer, Ultrafast transient generation of spin-density-wave order in the normal state of $\mathrm{BaFe}_{2} \mathrm{As}_{2}$ driven by coherent lattice vibrations, Nat. Mater. 11, 497 (2012).

[21] R. Mankowsky, A. Subedi, M. Först, S. O. Mariager, M. Chollet, H. Lemke, J. Robinson, J. Glownia, M. Minitti, A. Frano, M. Fechner, N. A. Spaldin, T. Loew, B. Keimer, A. Georges, and A. Cavalleri, Nonlinear lattice dynamics as a basis for enhanced superconductivity in $\mathrm{YBa}_{2} \mathrm{Cu}_{3} \mathrm{O}_{6.5}$, Nature 516, 71 (2014).

[22] G. Nieva E. Osquiguil, J. Guimpel, M. Maenhoudt, B. Wuyts, Y. Bruynseraede, M. B. Maple, and Ivan K. Schuller, Photoinduced enhancement of superconductivity, Appl. Phys. Lett. 60, 2159 (1992).

[23] R. D. Averitt, G. Rodriguez, A. I. Lobad, J. L. W. Siders, S. A. Trugman, and A. J. Taylor, Nonequilibrium superconductivity and quasiparticle dynamics in $\mathrm{YBa}_{2} \mathrm{Cu}_{3} \mathrm{O}_{7-\delta}$, Phys. Rev. B 63, 140502(R), (2001).

[24] W. Hu, S. Kaiser, D. Nicoletti, C. R. Hunt, I. Gierz, M. C. Hoffmann, M. Le Tacon, T. Loew, B. Keimer, and A. Cavalleri, Optically enhanced coherent transport in $\mathrm{YBa}_{2} \mathrm{Cu}_{3} \mathrm{O}_{6.5}$ by ultrafast redistribution of interlayer coupling, Nat. Mater. 13, 705 (2014).

[25] S. Kaiser, C. R. Hunt, D. Nicoletti, W. Hu, I. Gierz, H. Y. Liu, M. Le Tacon, T. Loew, D. Haug, B. Keimer, and A. Cavalleri. Optically induced coherent transport far above $T_{c}$ in underdoped $\mathrm{YBa}_{2} \mathrm{Cu}_{3} \mathrm{O}_{6+\delta}$, Phys. Rev. B 89, 184516 (2014).

[26] M. Först, R. I. Tobey, H. Bromberger, S. B. Wilkins, V. Khanna, A. D. Caviglia, Y.-D. Chuang, W. S. Lee, W. F. Schlotter, J. J. Turner, M. P. Minitti, O. Krupin, Z. J. Xu, J. S. Wen, G. D. Gu, S. S. Dhesi, A. Cavalleri, and J. P. Hill, Melting of charge stripes in vibrationally driven $\mathrm{La}_{1.875} \mathrm{Ba}_{0.125} \mathrm{CuO}_{4}$ : Assessing the respective roles of electronic and lattice order in frustrated superconductors, Phys. Rev. Lett. 112, 157002 (2014).

[27] M. Born and E. Wolf, Principles of Optics, 7th ed. (Cambridge University Press, Cambridge, 1999), p. 54.

[28] See Supplemental Material at http://link.aps.org/supplemental/ 10.1103/PhysRevB.91.020505 for a description of how the optical response was modeled.

[29] J. Fink, V. Soltwisch, J. Geck, E. Schierle, E. Weschke, and B. Büchner, Phase diagram of charge order in $\mathrm{La}_{1.8-x} \mathrm{Eu}_{0.2} \mathrm{Sr}_{x} \mathrm{CuO}_{4}$ from resonant soft x-ray diffraction, Phys. Rev. B 83, 092503 (2011).

[30] J. H. Kim, H. S. Somal, M. T. Czyzyk, D. van der Marel, A Wittlin, A. M. Gerrits, V. H. M. Duijn, N. T. Hien, and A. A. Menovsky, Strong damping of the $c$-axis plasmon in high- $T_{c}$ cuprate superconductors, Physica C 247, 297 (1995).

[31] D. van der Marel and A. A. Tsvetkov, Transverse-optical Josephson plasmons: Equations of motion, Phys. Rev. B 64, 024530 (2001).

[32] An additional small, flat increase in $\sigma_{1}(\omega)$ may be the result of either a slight quasiparticle heating or a phonon reshaping, either of which primarily modifies frequencies beyond our $\mathrm{THz}$ range. Including additional terms to account for this effect does not impact the best-fit values of $\omega_{P}$ or $\Gamma$ so they are neglected here. 
[33] L. S. Bilbro R. Valdés Aguilar, G. Logvenov, O. Pelleg, I. Božović and N. P. Armitage, Temporal correlations of superconductivity above the transition temperature in $\mathrm{La}_{2-x} \mathrm{Sr}_{x} \mathrm{CuO}_{4}$ probed by terahertz spectroscopy, Nat. Phys. 7, 298 (2011).

[34] D. van der Marel, H. J. A. Molegraaf, J. Zaanen, Z. Nussinov, F. Carbone, A. Damascelli, H. Eisaki, M. Greven, P. H. Kes, and M. Li, Quantum critical behaviour in a high- $T_{c}$ superconductor, Nature (London) 425, 271 (2003).

[35] C. C. Homes, M. Hücker, Q. Li, Z. J. Xu, J. S. Wen, G. D. Gu, and J. M. Tranquada, Determination of the optical properties of $\mathrm{La}_{2-x} \mathrm{Ba}_{x} \mathrm{CuO}_{4}$ for several dopings, including the anomalous $x=1 / 8$ phase, Phys. Rev. B 85, 134510 (2012).

[36] S. Petit, A. H. Moudden, B. Hennion, A. Vietkin, and A. Revcolevischi, Spin dynamics study of $\mathrm{La}_{2-x} \mathrm{Sr}_{x} \mathrm{CuO}_{4}$ by inelastic neutron scattering, Physica B 234-236, 800 (1997).

[37] C. H. Lee and K. Yamada, Spin pseudogap in $\mathrm{La}_{2-x} \mathrm{Sr}_{x} \mathrm{CuO}_{4}$ studied by neutron scattering, Phys. Rev. B 67, 134521 (2003).

[38] M. Kofu, S.-H. Lee, M. Fujita, H.-J. Kang, H Eisaki, and K. Yamada, Hidden quantum spin-gap state in the static stripe phase of high-temperature $\mathrm{La}_{2-x} \mathrm{Sr}_{x} \mathrm{CuO}_{4}$ superconductors, Phys. Rev. Lett. 102, 047001 (2009).
[39] J. M. Tranquada, G. D. Gu, M. Hücker, Q. Jie, H.-J. Kang, R. Klingeler, Q. Li, N. Tristan, J. S. Wen, G. Y. Xu, Z. J. $\mathrm{Xu}$, J. Zhou, and M. v. Zimmermann, Evidence for unusual superconducting correlations coexisting with stripe order in $\mathrm{La}_{1.875} \mathrm{Ba}_{0.125} \mathrm{CuO}_{4}$, Phys. Rev. B 78, 174529 (2008).

[40] H.-J. Grafe, N. J. Curro, B. L. Young, A. Vyalikh, J. Vavilova, G. D. Gu, M. Hücker, and B. Büchner, Charge order and low frequency spin dynamics in lanthanum cuprates revealed by nuclear magnetic resonance, Eur. Phys. J. Spec. Top. 188, 89 (2010).

[41] Eduardo Fradkin, Steven A. Kivelson, and John M. Tranquada, Theory of intertwined orders in high temperature superconductors, arXiv:1407.4480.

[42] L. E. Hayward, David G. Hawthorn, R. G. Melko, and Subir Sachdev, Angular fluctuations of a multicomponent order describe the pseudogap of $\mathrm{YBa}_{2} \mathrm{Cu}_{3} \mathrm{O}_{6+x}$, Science 343, 1336 (2014).

[43] P. A. Lee, Amperean pairing and the pseudogap phase of cuprate superconductors, Phys. Rev. X 4, 031017 (2014).

[44] R. A. Kaindl, M. A. Carnahan, D. S. Chemla, S. Oh, and J. N. Eckstein, Dynamics of cooper pair formation in $\mathrm{Bi}_{2} \mathrm{Sr}_{2} \mathrm{CaCu}_{2} \mathrm{O}_{8+x}$, Phys. Rev. B 72, 060510(R) (2005). 